Journal of Engineering and Applied Sciences 14 (Special Issue 5): 8970-8973, 2019

ISSN: 1816-949X

(C) Medwell Journals, 2019

\title{
Study the Calibration of Bending Losses and Critical Radius of Curvature for Different Light Sources in Single-Mode Fibers
}

\author{
Wajeha Abdle Diam \\ Department of Laser Physics, Collage of Science for Women, University of Babylon, Iraq
}

\begin{abstract}
: the bending losses rise greatly of the longer wavelengths, inspite of the dependence of the wavelength is extremely strongly on the oscillatory because of the overlap with the reflected lightin the coating boundary or cladding as well as from the outer of the coating surface. The growing of bending losses at longer frequently of the wavelengths defines as the suitable range of the wavelength for the single-mode fiber. An experimental investigation was made of the bending losses and the calibration losses in a single-mode fiber using two types of different light sources that were the pure red laser diode with range of $635 \mathrm{~nm}$ as wavelength and the infrared light-emitting diode operate between $940-950 \mathrm{~nm}$. The measurements were carried out at the Communication and Postal Service, Al-Hillah-Babylon-Iraq. the bending losses, the calibration losses and the curvature critical radius were considered in this study. the results were contrasted with the theoretical data in order to investigate out that the both finding were very close.
\end{abstract}

$\underline{\text { Keywords: Bending losses, cladding, critical radius, single-mode fibre, laser diode }}$

\section{INTRODUCTION}

The technology of optical fiber that present the single-mode fiber as an optical fiber. This produce to transmit the single wave or the mode of light essentially a carrier as well as utilize for the long distance of the signal transmission (Zendehnam et al., 2010). Whereas, the multi mode fiber applies in the short distances. The cable of fiber optic consist of four parts Core, Cladding, Coating (buffer) and Jacket, as shown in Fig. 1. The suitable wavelength light is usually between $850 \mathrm{~nm}$ and $1610 \mathrm{~nm}$ (Nakajima et al., 2010). The core of the fiber was manufactured from high purity silica glass, whereas, the cladding that around the core is made from the glass with a lower index of refraction than the core, in order to ensure that the total internal reflection (Matsui et al., 2011; Zahra'a, 2014).

As ingle-mode fiber customarily consist of an arrow core of $8-9 \mu \mathrm{m}$ in diameter that yields only a single mode of light to propagate (Martins et al., 2009; Zahra'a, 2014). The narrowness diameter of the core leads to a lower number of internal reflections and minimizes optical dispersion, producing in faster signal propagation and lower optical dispersion loss. This allows wide signal b and width s (Zheng et al., 2014; Al Timimi et al., 2012). Bending losses is considered a commonly faced the problem in fiber optics that illustrate further propagation losses when the yare bent. That losses are raised very fast when the radius of certain critical bend is got (Watekar et al., 2009).

As is well-known in the core, the macro-bending can significantly increase the losses in fibers through the optical signal transmission because of leaving the light in to cladding. That commands to the defect in the mechanism of transmission (Saitoh et al., 2009; Ulrich et al., 1980). Generally, the selosses can be very significant when the critical radius value is reached. This critical radius is considerably larger than $10 \mathrm{~cm}$ of the single-mode fibers through the large mode are as in comparison with the small mode areas fibers (Nakajima et al., 2010; Nakajima et al., 2011).

In the optical fiber, the wave front should be perpendicular to the direction of propagation, when it is bends in the fiber. the signal transmitted in cladding is slower than in core. This may leads to losing in the signal transmitted energy into the optical fiber (Wu et al., 2011). therefore, the speed of light must be raise in the cladding to avoid that as that shown in Fig. 2. The light is lost from the core to the cladding when the wave incident angle in the core-cladding interface don't reach to the total internal reflection critical angle $(\theta \mathrm{c})$ (Ulrich et al., 1980; Makouei et al., 2007), as shown in Fig. 3.

The radiation attenuation coefficient $\left(\alpha_{r}\right)$ described the losses, where $\left(\alpha_{\mathrm{r}}\right)$ given by the Eq. 1:

$$
\alpha_{\mathrm{r}}=\mathrm{C}_{1} \mathrm{e}^{-\mathrm{C}_{2} R}
$$

where, $\mathrm{R}$ is a radius of curvature, $\mathrm{C}_{1}$ and $\mathrm{C}_{2}$ are constant (RCS) means the curvature critical radius of the single-mode fiber can be determined by:

$$
\mathrm{R}_{\mathrm{CS}}=20 \lambda /\left(\mathrm{n}_{1}-\mathrm{n}_{2}\right)^{3 / 2}\left(2.748-0.9966 \frac{\lambda}{\lambda \mathrm{c}}\right)^{-3}
$$

where, $\lambda_{c}$ is the cut-off wavelength for the single mode fiber.

A critical curvature of the radius is instantly proportional to the applied wavelength etc. Smaller wavelength guides to a smaller radius of curvature (Saitoh et al., 2010). 


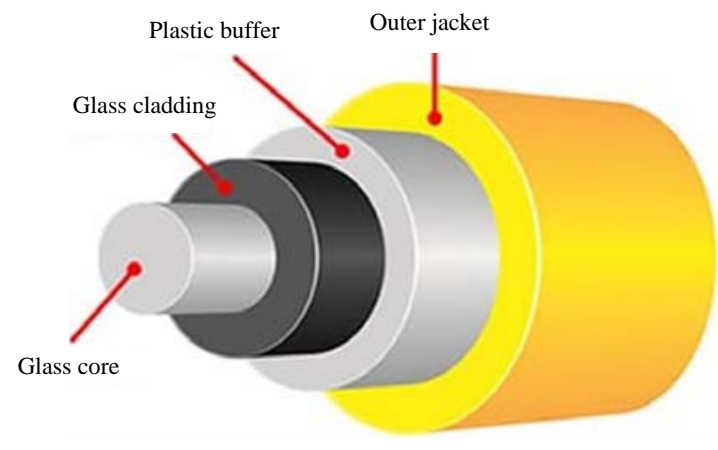

Fig. 1: Structure of a fiber optic cable

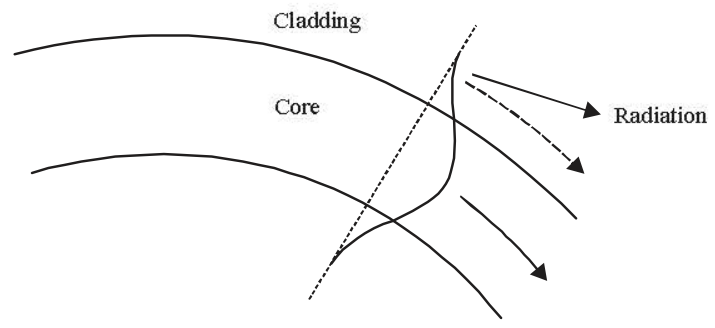

Fig. 2: Bending effect on transmission of radiation within the optical fibre

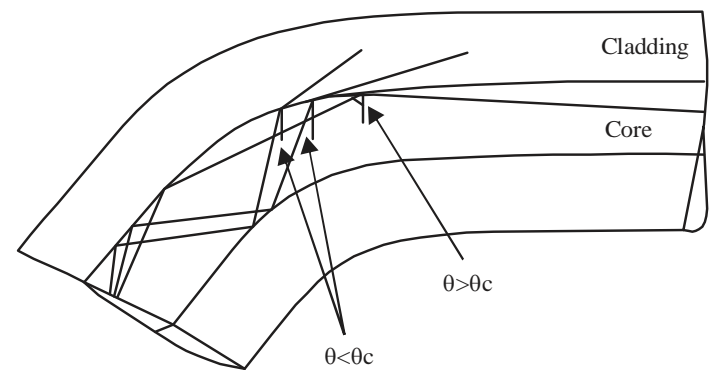

Fig. 3: Macro-bending schematic in an optical fibre

\section{MATERIALS AND METHOD}

The main specifications of used single-mode fiber were: $\lambda c$ was $1260 \mathrm{~nm}$ and the chromatic dispersion for the wavelength $1285 \mathrm{~nm}$ was $3 \mathrm{ps} /(\mathrm{nm} . \mathrm{km})$ while, for the wavelength $1322 \mathrm{~nm}$ was $22 \mathrm{ps} /(\mathrm{nm} . \mathrm{km})$.

After setup, a single-mode fiber, $4 \mathrm{~m}$, is the connection between the transmitter and the receiver, where the data transmission, which are numbers, characters and text message, are achieved for two different light sources. The first light source was infrared light-emitting diode operate between 940-950 nm model (COM-09349) which had high-density irradiation and greatest operation wavelength. The viewing angle $20^{\circ}$ and high reliability.

Pure red laser-diode was the second source, it is Model (RLD63NZC5) that work in the average wavelength $635 \mathrm{~nm}$ with $5 \mathrm{mw}$ output power, whereas, the process voltage and current are $2.2 \mathrm{~V}$ and $33 \mathrm{~mA}$, respectively. The measurements of the bending losses of curvature radius for both sources collected applying a power meter model, corning OM-610.

\section{RESULTS AND DISCUSSION}

The practical calibration of bending losses measurements of radius of curvature for infrared light-emitting diode equals to $-42.67 \mathrm{db}$ and for pure red laser diode was $-33.01 \mathrm{db}$.

The data of bending losses characterized applying the single mode optical fiber for infrared light-emitting diode operate between $940-950 \mathrm{~nm}$ showing in Table 1 and Fig. 4, where decreasing the radius of curvature leads to reduction in the output power, but with in certain of the curvature value, where the critical radius is $0.25 \mathrm{~mm}$ and the output power is cut off, whereas, applying the formulation (2) resulted a the theoretical critical radius equal $0.23 \mathrm{~mm}$.

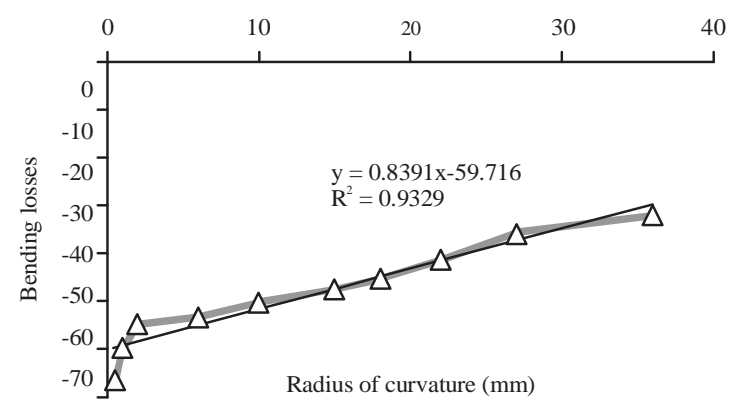

Fig. 4: Bending losses for infrared light-emitting diode operate between $940-950 \mathrm{~nm}$ source in single mode optical fibre 


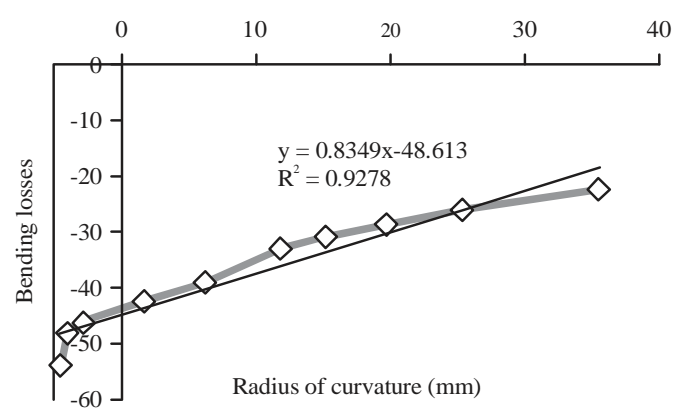

Fig. 5: Bending losses for pure red laser diode $(635 \mathrm{~nm})$ source in single mode optical fiber

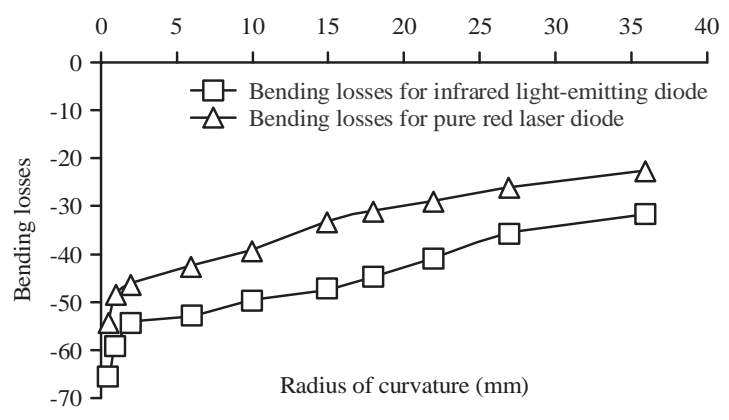

Fig. 6: Comparison of bending losses for pure red laser diode $(635 \mathrm{~nm})$ source and infrared light-emitting diode source in single mode optical fibre

Table 1: Bending losses for infrared light-emitting diode operate between $940-950 \mathrm{~nm}$ source in single mode optical fibre

\begin{tabular}{lc}
\hline Radius of curvature (mm) & Bending losses \\
\hline 0.5 & -65.67 \\
1 & -59.16 \\
2 & -54.43 \\
6 & -52.75 \\
10 & -49.78 \\
15 & -47.15 \\
18 & -44.75 \\
22 & -40.85 \\
27 & -35.67 \\
36 & -31.58 \\
\hline
\end{tabular}

Table 2: Bending losses for pure red laser diode $(635 \mathrm{~nm})$ source in single mode optical fiber

\begin{tabular}{lc}
\hline Radius of curvature $(\mathrm{mm})$ & Bending losses \\
\hline 0.5 & -54.02 \\
1 & -48.22 \\
2 & -46.18 \\
6 & -42.47 \\
10 & -39.05 \\
15 & -33.15 \\
18 & -30.89 \\
22 & -28.74 \\
27 & -26.14 \\
36 & -22.47 \\
\hline
\end{tabular}

The bending losses for pure red laser diode $(635 \mathrm{~nm})$ was characterized through the single mode optical fiber was shown in Table 2 and Fig. 5, where,
0.21 and $0.12 \mathrm{~mm}$ are the practical and theoretical curvature critical radius, respectively.

According to results from both sources, the results exhibited the high value of the curvature critical radius if increasing presented in the wavelength that matched the behaviour by Eq. 2. Figure 6 demonstrates the differences in the results between both sources. Where the measured results exhibited very close during the comparison between it. Meanwhile, this small different in the results related to the several factors such as, the properties of the optical fiber, which is linked to the loss of the calibration between the fiber and light sources (Zheng et al., 2014; Saitoh et al., 2009; Kashiwagi et al., 2012).

\section{CONCLUSION}

In this study, the infrared range was achieved when the wavelength was increased and the curvature critical radius of was high. That match the investigation of Eq. 2 .

When the critical radius was $0.25 \mathrm{~mm}$, the source was cut and the entire signal from infrared Light-Emitting Diode operate between 940 and $950 \mathrm{~nm}$, also when the entire signal from pure red laser diode was $635 \mathrm{~nm}$ the $\mathrm{n}$ the source was cut when the critical radius was $0.21 \mathrm{~mm}$.

\section{REFERENCES}

Al Timimi, Z., M.S. Jaafar, M.Z.M. Jafri, H.A.A. Houssein, F.H. Mustafa and G.A. Al-Dahash, 2012. The influence of low power laser energy on red blood cell and platelets (in vitro). Proceedings of the International Conference on Bioscience, Biochemistry and Pharmaceutical Sciences, February 11-12, 2012, Penang, Malaysia, pp: 12-14.

Kashiwagi, M., K. Saitoh, K. Takenaga, S. Tanigawa, S. Matsuo and M. Fujimaki, 2012. Effectively single-mode all-solid photonic bandgap fiber with large effective area and low bending loss for compact high-power all-fiber lasers. Opt. Express, 20: 15061-15070.

Makouei, S., M.S. Oskouei and A. Rostami, 2007. Study of bending loss and mode field diameter in depressed inner core triple-clad single-mode optical fibers. Opt. Commun., 280: 58-67.

Martins, A., A.M. Rocha, B. Neto, A.L.J. Teixeira and M. Facao et al., 2009. Modeling of bend losses in single-mode optical fibers. Proceedings of the 7th Conference on Telecommunications, May 3-5, 2009, Santa Maria da Feira, Portugal.

Matsui, T., T. Sakamoto, K. Tsujikawa, S. Tomita and M. Tsubokawa, 2011. Single-mode photonic crystal fiber design with ultralarge effective area and low bending loss for ultrahigh-speed WDM transmission. J. Lightwave Technol., 29: 511-515. 
Nakajima, K., T. Matsui, S. Aozasa and T. Shimizu, 2011. Single-mode hole-assisted fiber with low bending loss characteristics. Proceedings of the IEEE Winter Topicals, January 10-12, 2011, Keystone, CO., USA., pp: 175-176.

Nakajima, K., T. Shimizu, T. Matsui, C. Fukai and T. Kurashima, 2010. Single-mode hole-assisted fiber as a bending-loss insensitive fiber. Opt. Fiber Technol., 16: 392-398.

Saitoh, K., T. Murao, L. Rosa and M. Koshiba, 2010. Effective area limit of large-mode-area solid-core photonic bandgap fibers for fiber laser applications. Opt. Fiber Technol., 16: 409-418.

Saitoh, K., Y. Tsuchida and M. Koshiba, 2009. Design of effectively single-mode leakage channel fibers with large mode area and low bending loss. IEICE. Electron. Express, 6: 412-417.

Ulrich, R., S.C. Rashleigh and W. Eickhoff, 1980. Bending-induced birefringence in single-mode fibers. Opt. Lett., 5: 273-275.
Watekar, P.R., S. Ju and W.T. Han, 2009. Design and development of a trenched optical fiber with ultra-low bending loss. Opt. Express, 17: 10350-10363.

Wu, Q., Y. Semenova, B. Yan, Y. Ma, P. Wang, C. Yu and G. Farrell, 2011. Fiber refractometer based on a fiber Bragg grating and single-modemultimode-single-mode fiber structure. Opt. Lett., 36: 2197-2199.

Zahra'a, A.T., 2014. Exploration of additional mechanical phenomena of laser-tissue interaction contributes to the damage and elimination of the small blood vessels. Int. J. Scient. Eng. Res., 5: 672-675.

Zendehnam, A., M. Mirzaei, A. Farashiani and L.H. Farahani, 2010. Investigation of bending loss in a single-mode optical fibre. Pramana, 74: 591-603.

Zheng, S., G. Ren, Z. Lin, W. Jian and S. Jian, 2014. Design and analysis of novel multilayer-core fiber with large mode area and low bending loss. Opt. Commun., 315: 317-323. 